\title{
EFFECTS OF CHEMICAL REACTION ON MAGNETO-MICROPOLAR FLUID FLOW FROM A RADIATIVE SURFACE WITH VARIABLE PERMEABILITY
}

\author{
B.K. SHARMA* \\ Department of Mathematics \\ Department of Civil Engineering \\ Birla Institute of Technology and Science \\ Pilani Rajasthan, INDIA \\ E-mail: bhupen_1402@yahoo.co.in \\ A.P. SINGH \\ Department of Civil Engineering \\ Birla Institute of Technology and Science \\ Pilani Rajasthan, INDIA \\ K. YADAV and R.C. CHAUDHARY \\ Department of Mathematics \\ University of Rajasthan, Jaipur, INDIA
}

\begin{abstract}
This paper presents a study of a hydromagnetic free convection flow of an electrically conducting micropolar fluid past a vertical plate through a porous medium with a heat source, taking into account the homogeneous chemical reaction of first order. A uniform magnetic field has also been considered in the study which acts perpendicular to the porous surface of the above plate. The analysis has been done by assuming varying permeability of the medium and the Rosseland approximation has been used to describe the radiative heat flux in the energy equation. Numerical results are presented graphically in the form of velocity, micro- rotation, concentration and temperature profiles, the skin-friction coefficient, the couple stress coefficient, the rate of heat and mass transfers at the wall for different material parameters. The study clearly demonstrates how a chemical reaction influences the above parameters under given conditions.
\end{abstract}

Key words: chemical reaction, micro-polar fluid, micro-rotation, magneto-hydrodynamics, heat and mass transfer.

MSC 2000: 80A20, 76R10, 76S05.

\section{Introduction}

In many engineering applications such as separation processes in chemical engineering, heat and mass transfer characteristics have been used widely. For example, evaporation, condensation, distillation, rectification processes in fluids condensing or boiling at a solid surface play a decisive role (Baehr and Stephan, 1998). In order to study the theory of micropolar fluids, Eringen (1964) developed a simple theory which includes the effect of local rotary inertia, the couple stress and the inertial spin. This theory is expected to be useful in analyzing the behavior of non-Newtonian fluids. Eringen (1966) also developed the theory of micropolar fluids for the cases where only microrotational effects and microrotational inertia exist. Eringen (1972) extended the theory of thermo-micropolar fluids and derived the constitutive law for fluids

\footnotetext{
* To whom correspondence should be addressed
} 
with microstructure. This general theory of micropolar fluids deviates from that of Newtonian fluids by adding two new variables to the velocity. These variables are micro-rotation that is spin and microinertia tensor describing the distributions of atoms and molecules inside the microscopic fluid particles. The theory may be applied to explain the phenomenon of the flow of colloidal fluids, liquid crystals, polymeric suspensions, animal blood etc. In view of Lukaszewicz (1999), micropolar fluids represent those fluids which consist of randomly oriented particles suspended in a viscous medium. Several authors have studied the characteristics of the boundary layer flow of a micropolar fluid under different boundary conditions. An excellent review of micropolar fluids and their applications was given by Ariman et al. (1973). Gorla (1992) also discussed the steady state heat transfer in a micropolar fluid flow over a semi-infinite plate. Rees and Pop (1998) studied free convection boundary layer flow of a micropolar fluid from a vertical flat plate. Takhar and Soundalgekar $(1980 ; 1985)$ studied the flow and heat transfer of a micropolar fluid past a porous plate. Further, they $(1983 ; 1985)$ discussed these problems for the flow past a continuously moving porous plate. A micropolar fluid flow over a horizontal plate with surface mass transfer was presented by Yucel (1989). Gorla et al. (1998; 1995) investigated further the concept of natural convection from a heated vertical plate in a micropolar fluid.

Flows of fluids through porous media are of principal interest because they are quite prevalent in nature. Such flows have attracted the attention of a number of scholars due to their applications in many branches of science and technology, viz., in the fields of agriculture engineering to study the underground water resources, seepage of water in river beds, in petroleum technology to study the movement of natural gas, oil, and water through oil reservoirs, in chemical engineering for filtration and purification processes. Hiremath and Patil (1993) studied the effect of free convection currents on the oscillatory flow of a polar fluid through a porous medium, which is bounded by a vertical plane surface of constant temperature.

The problem of flow and heat transfer for a micropolar fluid past a porous plate embedded in a porous medium has been of great interest in engineering studies such as oil exploration, thermal insulation, etc. Raptis and Takhar (1999) considered a micropolar fluid flow through a porous medium. Fluctuating heat and mass transfer of three-dimensional flow through a porous medium with variable permeability was discussed by Sharma et al. (2007).

Hydromagnetic convection with heat and mass transfer has been studied due to its importance in the design of magnetohydrodynamics (MHD) generators and accelerators in geophysics, astrophysics, nuclear power reactors and so on. The interest in these new problems generates from their importance in liquid metals, electrolytes and ionized gases. The unsteady hydromagnetic free convection flow of Newtonian and polar fluids was investigated by Helmy (1998). Chaudhary and Sharma (2006) considered combined heat and mass transfer by laminar mixed convection flow from a vertical surface with induced magnetic field. Hydromagnetic unsteady mixed convection and mass transfer flow past a vertical porous plate immersed in a porous medium was investigated by Sharma and Chaudhary (2008). El-Hakien et al. (1999) studied the effects of viscous flow and Joule heating on the MHD-free convection flow with variable plate temperature in a micropolar fluid. El-Amin (2001) considered the MHD free-convection and mass transfer flow in a micropolar fluid over a stationary vertical plate with constant suction. Kim (2001) investigated the unsteady free convection flow of a micropolar fluid past a vertical plate embedded in a porous medium and extended his work (2004) to study the effects of heat and mass transfer in the MHD micropolar fluid flow past a vertical moving plate. Analytical studies on the MHD flow of a micropolar fluid over a vertical porous plate were presented by Kim and Lee (2003) and Helmy et al. (2002).

Combined heat and mass transfer problems with chemical reaction are of importance in many processes and have, therefore, received a considerable amount of attention in recent years. In processes such as drying, evaporation at the surface of water body, energy transfer in wet cooling tower and the flow in a desert cooler, heat and mass transfer occur simultaneously. Chemical reactions can be codified as either homogeneous or heterogeneous processes. A homogeneous reaction is one that occurs uniformly through a given phase. In contrast, a heterogeneous reaction takes place in a restricted region or within the boundary of a phase. A reaction is said to be of first order, if the rate of reaction is directly proportional to the concentration itself which has many applications in different chemical engineering processes and other industrial applications such as polymer production, manufacturing of ceramics or glassware and food 
processing (Cussler, 1998). Das et al. (1994) considered the effects of first order chemical reaction on the flow past an impulsively started infinite vertical plate with constant heat flux and mass transfer. Muthucumarswamy and Ganesan (2001) and Muthucumarswamy (2002) studied a first order homogeneous chemical reaction on flow past an infinite vertical plate.

In the above mentioned studies the effects of heat sources/sinks and radiation have not been considered. Many processes in new engineering areas occur at high temperature and knowledge of heat transfer becomes imperative for the design of the pertinent equipment. Nuclear power plants, gas turbines and various propulsion devices for aircraft, missiles, satellites, and space vehicles are examples of such engineering areas. Kandasamy et al. (2005) discussed the heat and mass transfer effect along a wedge with heat source and concentration in the presence of suction/injection taking into account the chemical reaction of first order. Sharma et al. (2006a; 2006b) reported on the radiation effect with simultaneous thermal and mass diffusion in the MHD mixed convection flow from a vertical surface. Perdikis and Repatis (1996) illustrated the heat transfer of a micropolar fluid in the presence of radiation. Raptis (1998) studied the effect of radiation on the flow of a micropolar fluid past a continuously moving plate. Elbashbeshby and Bazid (2000) and Kim and Fedorov (2003) reported on the radiation effects on the mixed convection flow of a micropolar fluid. Moreover, when the radiative heat transfer takes place, the fluid involved can be electrically conducting in the sense that it is ionized owing to high operating temperature. The heat and mass transfer in a magneto hydrodynamic micropolar fluid flow through a porous medium under different physical conditions was examined by Ibrahim et al. (2004). Since the flow past a continuously moving plate has many applications in manufacturing processes, Rahman and Sattar (2006) analyzed the MHD convective flow of a micropolar fluid past a continuously moving vertical porous plate in the presence of heat generation/absorption. The process of fusing of metals in an electrical furnace by applying a magnetic field and the process of cooling of the first wall inside a nuclear reactor containment vessel where the hot plasma is isolated from the wall by applying a magnetic field are examples of such fields where thermal radiation and magneto-hydrodynamics are correlative. This fact was taken into consideration by Aziz (2006) in his study on micropolar fluids. Sharma et al. $(2007 ; 2008)$ discussed the radiation effect on the free convective flow along a uniform moving porous vertical plate in the presence of heat source/sink and transverse magnetic field. Recently, Chaudhary and Jain (2007) investigated the combined heat and mass transfer in a magneto-micropolar fluid flow from a radiative surface with variable permeability. In the present analysis, it is proposed to study the effect of a first order homogeneous chemical reaction on a magneto-micropolar fluid flow past a radiative vertical plate through a porous medium with a heat source.

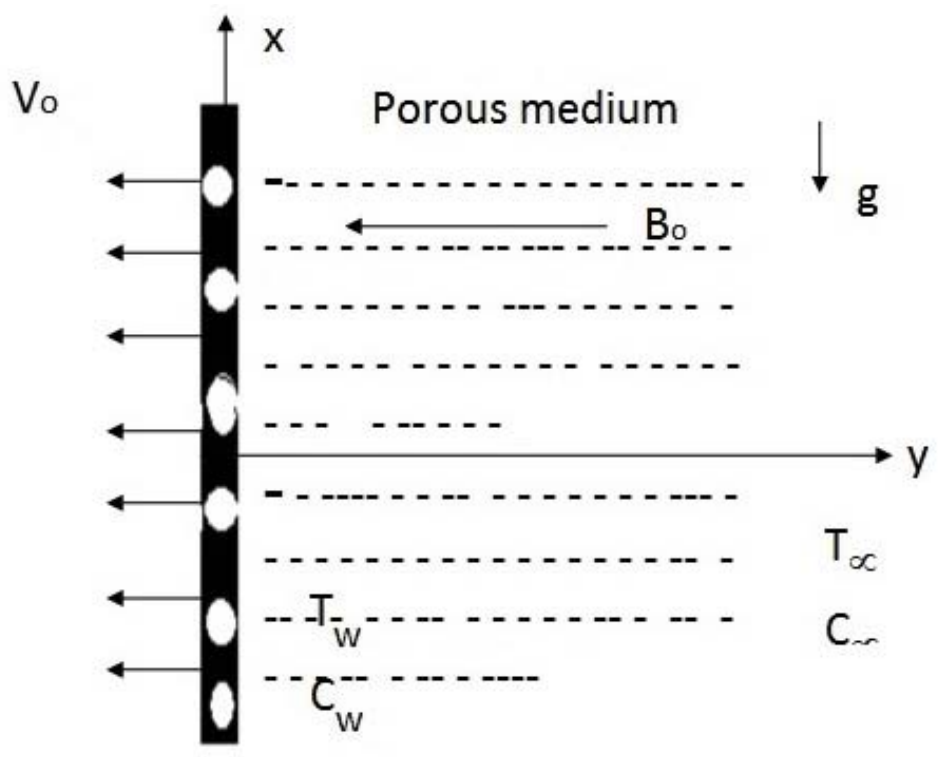

Fig.1. Physical model of the problem. 


\section{Mathematical formulation}

In this study, the two-dimensional flow of a micropolar fluid past a semi-infinite vertical plate embedded in a porous medium has been considered by taking the $x$-axis along the porous plate and the $y$-axis normal to it as shown in Fig.1. Due to the semi-infinite plane surface assumption, the flow variables are functions of $y$ and $t$ only. In order to derive the basic equations for the problem under investigation, the following assumptions have been made:

1. The fluid under consideration is viscous, incompressible and electrically conducting with constant physical properties.

2. The flow is unsteady, laminar and the magnetic field is applied perpendicular to the plate.

3. Viscous and Darcy's resistance terms are taken into account with variable permeability porous medium.

4. The magnetic Reynolds number is assumed to be small enough so that the induced magnetic field can be neglected.

5. The Hall Effect, Joule Heating, and viscous dissipation are all neglected in this study.

6. The fluid is considered to be a gray, absorbing-emitting but non-scattering medium and the Rosseland approximation is used to describe the radiative heat flux.

7. There is a first order chemical reaction between the diffusing species and the fluid.

8. It is also assumed that there is no applied voltage which implies the absence of an electric field.

Under these assumptions and introducing non-dimensional quantities, the governing equations for the flow are as follows

$$
\begin{aligned}
& \frac{\partial u}{\partial t}-\left(1+\in B e^{n t}\right) \frac{\partial u}{\partial y}=(1+B v) \frac{\partial^{2} u}{\partial y^{2}}+\mathrm{Gr} \theta+\mathrm{Gc} C+\left[M+\frac{1}{\alpha\left(1+A e^{n t}\right)}\right]+2 B v \frac{\partial \omega}{\partial y}, \\
& \frac{\partial \omega}{\partial t}-\left(1+\in B e^{n t}\right) \frac{\partial \omega}{\partial y}=\frac{1}{\varphi} \frac{\partial^{2} \omega}{\partial y^{2}} \\
& \frac{\partial \theta}{\partial t}-\left(1+\in B e^{n t}\right) \frac{\partial \theta}{\partial y}=\left(\frac{3 R+4}{3 R \operatorname{Pr}}\right) \frac{\partial^{2} \theta}{\partial y^{2}}+S \theta \\
& \frac{\partial C}{\partial t}-\left(1+\in B e^{n t}\right) \frac{\partial C}{\partial y}=\frac{1}{\mathrm{Sc}} \frac{\partial^{2} c}{\partial y^{2}}-K_{1} C
\end{aligned}
$$

where $\quad \varphi=\frac{\mu J^{*}}{\gamma}=\frac{2}{2+B v}$,

with the corresponding boundary conditions

$$
\begin{aligned}
& u=1, \quad \omega=-m \frac{\partial u}{\partial y}, \quad \theta=1+\varepsilon e^{n t}, \quad C=1+\varepsilon e^{n t} \text { at } \quad y=0, \\
& u \rightarrow 0, \quad \omega \rightarrow 0, \quad \theta \rightarrow 0, \quad C \rightarrow 0 \quad \text { as } \quad y \rightarrow \infty .
\end{aligned}
$$




\section{Solution for the problem}

In order to reduce the aforesaid system of partial differential equations to a system of ordinary differential equations in a dimensionless form, we may write the translational velocity, microrotation, temperature and concentration as

$$
\begin{aligned}
& u=u_{0}(y)+\in e^{n t t} u_{l}(y)+0\left(\epsilon^{2}\right), \\
& \omega=\omega_{0}(y)+\epsilon e^{n t} \omega_{l}(y)+0\left(\epsilon^{2}\right), \\
& \theta=\theta_{0}(y)+\in e^{n t t} \theta_{l}(y)+0\left(\epsilon^{2}\right), \\
& C=C_{0}(e)+\in e^{n t} C_{l}(y)+0\left(\epsilon^{2}\right) .
\end{aligned}
$$

By substituting the above Eqs (3.1) - (3.4) into Eqs (2.1) - (2.4), neglecting the coefficient of $0\left(\epsilon^{2}\right)$, we obtain the following pairs of equations for $\left(u_{0}, \omega_{0}, \theta_{0}, C_{0}\right)$ and $\left(u_{1}, \omega_{1}, \theta_{1}, C_{1}\right)$

$$
\begin{aligned}
& (1+B v) u_{0}^{\prime \prime}+u_{0}^{\prime}-\bar{M} u_{0}=-\mathrm{Gr} \theta_{0}-\mathrm{Gc} C_{0}-2 B v \omega_{0}^{\prime}, \\
& (1+B v) u_{1}^{\prime \prime}+u_{1}^{\prime}-(\bar{M}+n) u_{1}=-\operatorname{Gr} \theta_{1}-\mathrm{Gc} C_{1}-2 B v \omega_{1}^{\prime}-B u_{0}^{\prime}-\frac{A u_{0}}{\alpha^{2}}, \\
& \omega_{0}^{\prime \prime}+\varphi \omega_{0}^{\prime}=0, \\
& \omega_{1}^{\prime \prime}+\varphi \omega_{1}^{\prime}-\varphi n \omega_{1}=-B \varphi \omega_{0}^{\prime}, \\
& \theta_{0}^{\prime \prime}+b_{1} \theta_{0}^{\prime}-\operatorname{Sb} \theta_{1} \theta_{0}=0, \\
& \theta_{1}^{\prime \prime}+b_{1} \theta_{1}^{\prime}+b_{1}(S+n) \theta_{1}=-B b_{1} \theta_{0}^{\prime}, \\
& C_{0}^{\prime \prime}+\operatorname{Sc} C_{0}^{\prime}-K_{1} \operatorname{Sc} C_{o}=0, \\
& C_{1}^{\prime \prime}+\operatorname{Sc} C_{1}^{\prime}-\left(n+K_{1}\right) \operatorname{Sc} C_{1}=-B \operatorname{Sc} C_{0}^{\prime}
\end{aligned}
$$

where the primes denote differentiation with respect to $y$.

Here $\quad \bar{M}=M+\frac{1}{\alpha}, \quad b_{1}=\frac{3 R \operatorname{Pr}}{3 R+4}$.

The corresponding boundary conditions can be written as 


$$
\begin{aligned}
& u_{0}=1, \quad u_{1}=0, \quad \omega_{0}=-m u_{0}^{\prime}, \quad \omega_{1}=-m u_{1}^{\prime}, \quad \theta_{0}=1, \\
& \theta_{1}=0, \quad C_{0}=1, \quad C_{1}=0 \quad \text { at } \quad y=0, \\
& u_{0}=1, \quad u_{1}=1, \quad \omega_{0}=0, \quad \omega_{1}=0, \quad \theta_{0}=0, \\
& \theta_{1}=0, \quad C_{0}=0, \quad C_{1}=0 \quad \text { as } \quad y \rightarrow \infty .
\end{aligned}
$$

The solutions of Eqs (3.6) - (3.12) with the satisfying boundary conditions (3.13) and (3.14) are given by

$$
\begin{aligned}
& u_{0}(y)=j_{11} e^{-a_{5} y}-j_{12} e^{-a_{2} y}-j_{13} e^{-a_{3} y}+j_{14} L_{6} e^{-F_{1} y}, \\
& u_{1}=L_{11} e^{-d_{15} y}+d_{8} e^{-a_{1} y} d_{9} e^{-a_{2} y}+d_{10} e^{-a_{3} y}+ \\
& +d_{11} e^{-a_{5} y}+d_{12} e^{-F_{1} y}+d_{13} e^{-a_{4} y}+d_{14} L_{10} e^{-a_{10} y} \\
& \omega_{0}(y)=L_{6} e^{-F_{1} y} \\
& \omega_{1}(y)=L_{10} e^{-F_{3} y}+L_{9} e^{-F_{1} y} \\
& \theta_{0}(y)=e^{-a_{2} y} \\
& \theta_{1}(y)=c_{2} e^{-a_{2} y}+c_{3} e^{-a_{2} y} \\
& C_{0}(y)=e^{-a_{3} y} \\
& C_{1}(y)=f_{8} e^{-a_{4} y}+f_{5} e^{-a_{3} y}+f_{6} e^{-a_{2} y}+f_{7} e^{-a_{1} y} .
\end{aligned}
$$

The constants are not given for the sake of brevity.

\section{Results and discussion}

The MHD convective flow and mass transfer of an incompressible micropolar fluid along a semiinfinite vertical plate in a porous medium with a heat source and chemical reaction has been studied in preceding sections. In order to get a physical insight into the problem, numerical calculations for the distribution of the translational velocity, microrotation, temperature and concentration rate of heat transfer and mass transfer across the boundary layer for various values of the parameter have been done. The effects of the main controlling parameters as they appear in the governing equations are discussed in the current section. In this study, entire numerical calculations have been performed with $\varepsilon=0.01, n=1$ and $t=1$ while $\operatorname{Pr}, R$, Sc, Gr, Gc, $B v, M, \alpha, S, m, B, K_{l}$ and $A$ are varied over ranges, which are listed in the figure legends. In the absence of the chemical reaction and heat source, temperature profiles and concentration profiles have been analyzed they are in good agreement with the available results of Chaudhary and Jain (2007) as shown in Figs 2 and 4, respectively. 
Typical variations of the temperature profiles $\theta(y)$ along the span-wise coordinate $y$ are shown in Fig. 3 for different values of the Prandtl number $\left(\mathrm{Pr}=0.71\right.$, for air at $20^{\circ} \mathrm{C}$ and 1 atmospheric pressure, $\operatorname{Pr}=1.0$ for electrolytic solution, at $20^{\circ} \mathrm{C}$ and 1 atmospheric pressure), the radiation parameter $(R)$ and suction parameter $(B)$. The numerical results show that the temperature decreases with an increase in the Prandtl number. This is due to the fact that a fluid with a high Prandtl number has a relatively low thermal conductivity which results in the reduction of the thermal boundary layer thickness. Also, the figure indicates that the temperature reduces with an increase in the radiation parameter $(R)$, suction parameter $(B)$ and heat source for air while a reverse effect is observed for the electrolytic solution .

Figure 4 depicts the species concentration for different gases. The values of the Schmidt number $(\mathrm{Sc})$ are chosen to represent the most common diffusing chemical species like hydrogen $(\mathrm{Sc}=0.22)$, oxygen $(\mathrm{Sc}=0.66)$, and ammonia $(\mathrm{Sc}=0.78)$ at a temperature of $25^{\circ} \mathrm{C}$ and 1 atmospheric pressure. For $\mathrm{Sc}=0.22, B=0.1$ and $K 1=0$, the concentration profile is same as obtained by Chaudhary and Jain (2007). A comparison of curves in the figure shows a decrease in concentration distribution $C(y)$ with an increase in the Schmidt number because the smaller values of Sc are equivalent to increasing chemical molecular diffusivity $(D)$. Hence the concentration of the species is higher for small values of Sc and lower for larger values of Sc. The concentration profiles also decrease with an increase in the suction parameter $(B)$. There is a fall in the concentration due to increasing values of the chemical reaction parameter. This shows that the diffusion rates can be significantly altered by a chemical reaction. Both the temperature and the concentration profiles attain their maximum values at the wall and decrease exponentially with $y$ and finally tend to zero as $y \rightarrow \infty$.

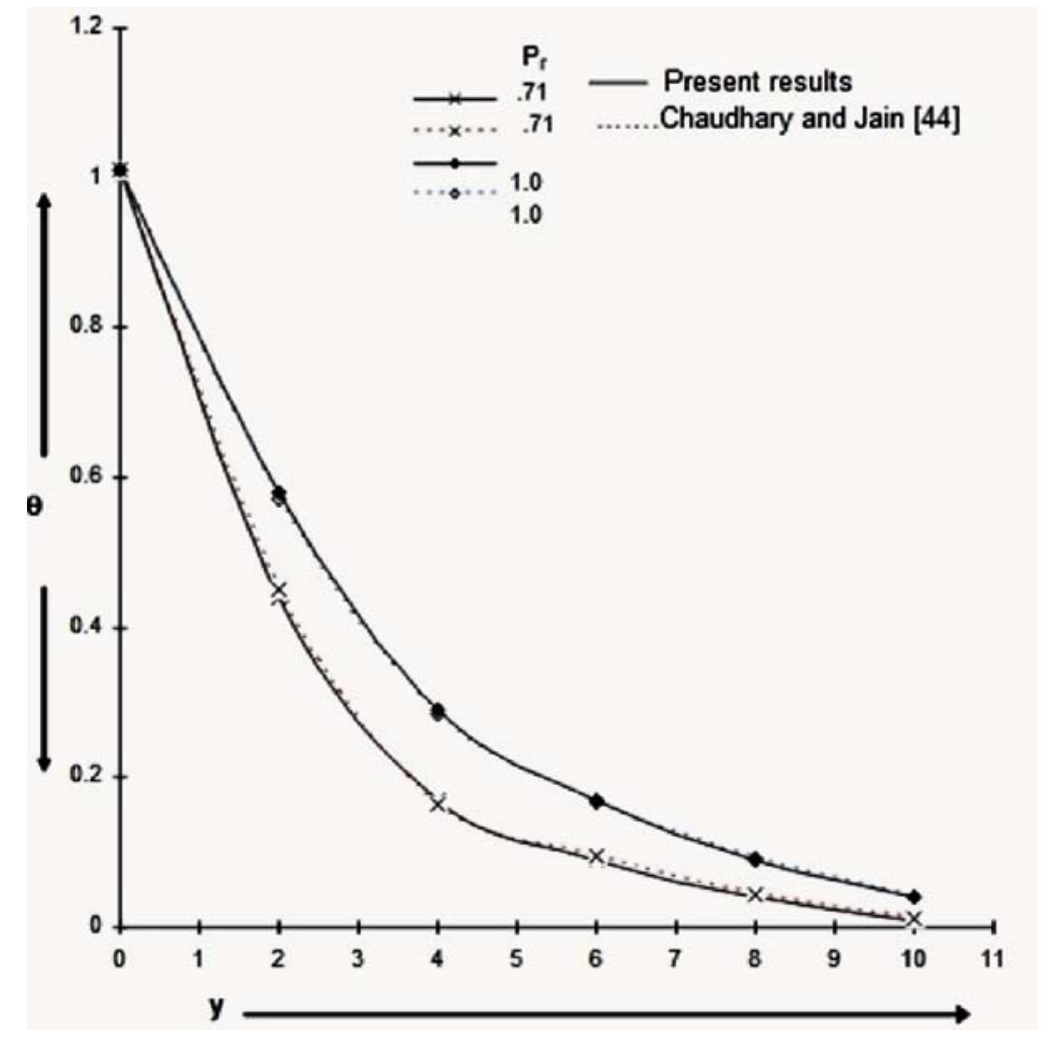

Fig.2. Temperature profiles for $B=0.1, R=1.0$ and $S=0$. 


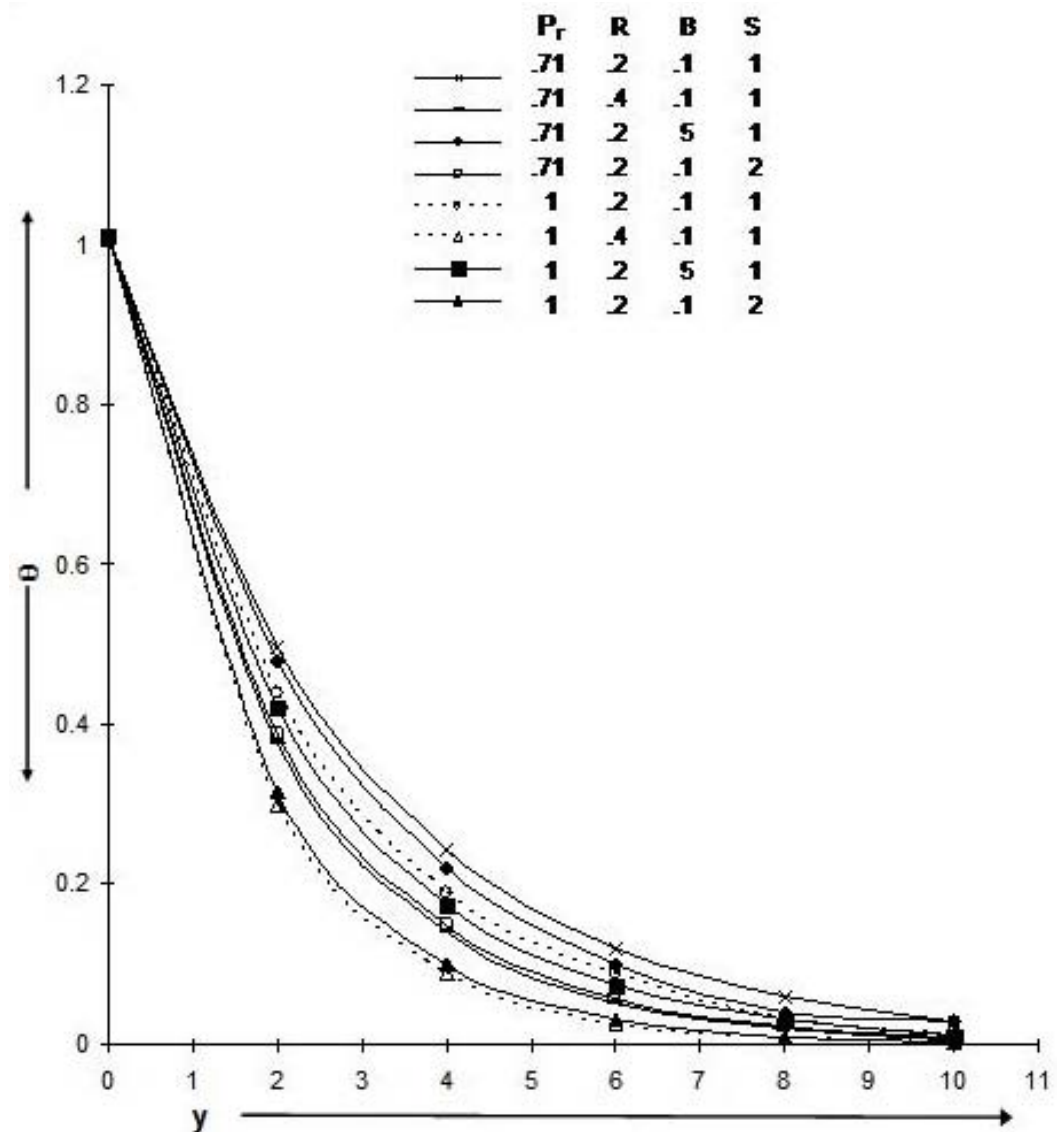

Fig.3. Temperature profiles for $\varepsilon=0.01, n=0.1, t=1$.

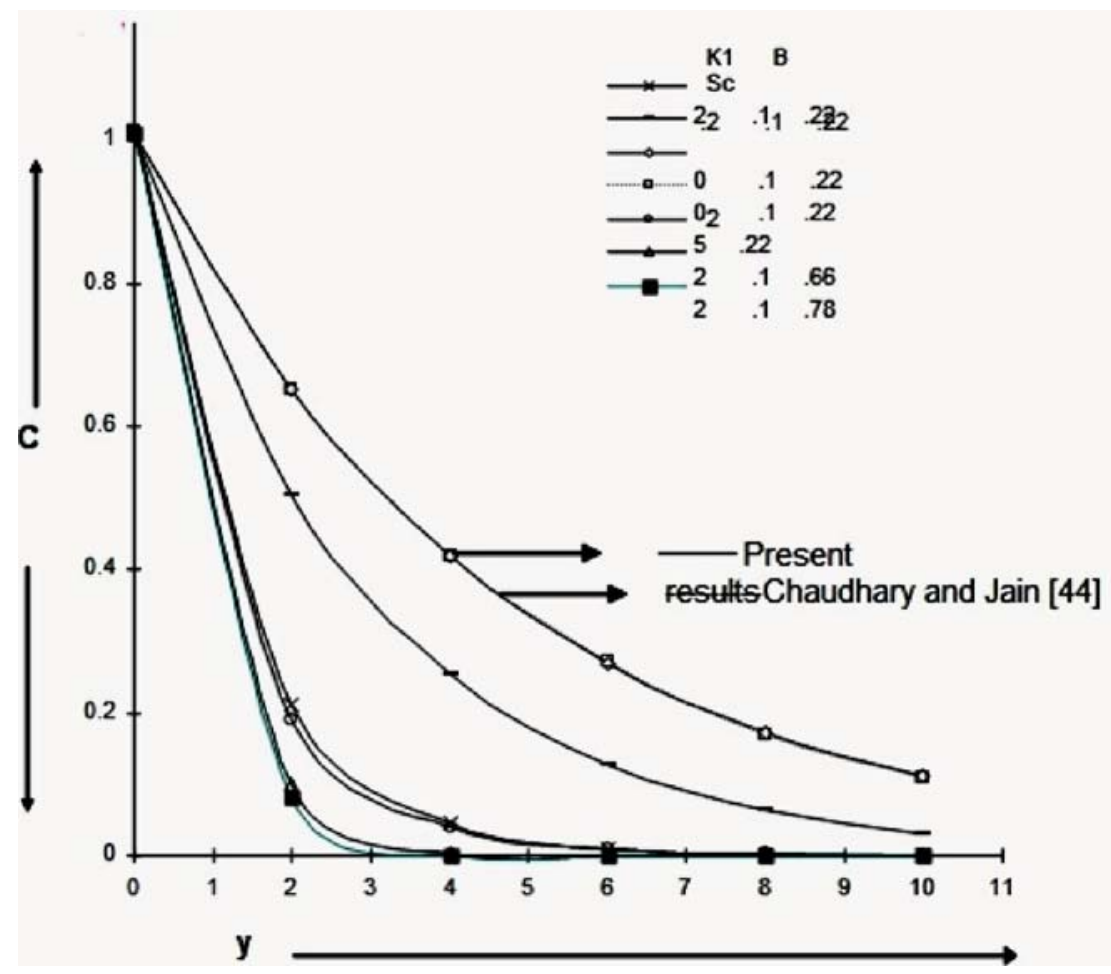

Fig.4. Concentration profiles for $\varepsilon=0.01, n=0.1, t=1$. 
For different values of the radiation parameter $(R)$, chemical reaction parameter $\left(K_{l}\right)$, viscosity ratio parameter $\left(B_{v}\right)$, permeability parameter $(\alpha)$, the translational velocity $u$ and microrotation profiles $\omega$ are plotted in Figs 5 and 6 , respectively. It is noteworthy that the velocity $u$ and the magnitude of angular velocity $\omega$ decrease as the radiation parameter $(R)$ increases. This result can be explained by the fact that a decrease in the radiation parameter $R=k a_{R} / 4 \sigma^{*} T_{\infty}^{3}$ for a given $k$ and $T_{\infty}$, means a decrease in the Rosseland radiation absorbtivity $\left(a_{R}\right)$. In view of Eqs (A5) and (A6), it is concluded that the divergence of the radiative heat flux $\partial q_{r} / \partial y^{*}$ increases as $a_{R}$ decreases and this means that the rate of radiative heat transferred to the fluid increases and consequently the fluid temperature (see Fig.3) and hence the velocity of its particles also increases. Moreover, figures reveal that on increasing the values of the permeability parameter $(\alpha)$ the profiles of $u$ and the magnitude of $\omega$, across the boundary layer, tend to increase. It is noted that translational velocity increases with decreasing the chemical reaction parameter, while, a reverse effect is observed for microrotation profile. The velocity distribution decreases with increasing $B_{v}$. The phenomenon reflects the the fact that the effect of increase in the value of $B_{v}$ will result in an enhancement of the total viscosity in the fluid flow because $B_{v}$ is directly proportional to vortex viscosity which makes the fluid flow more viscous and so weakens the convection currents. In addition, the magnitude of $\omega$ increases as $B_{v}$ increases.

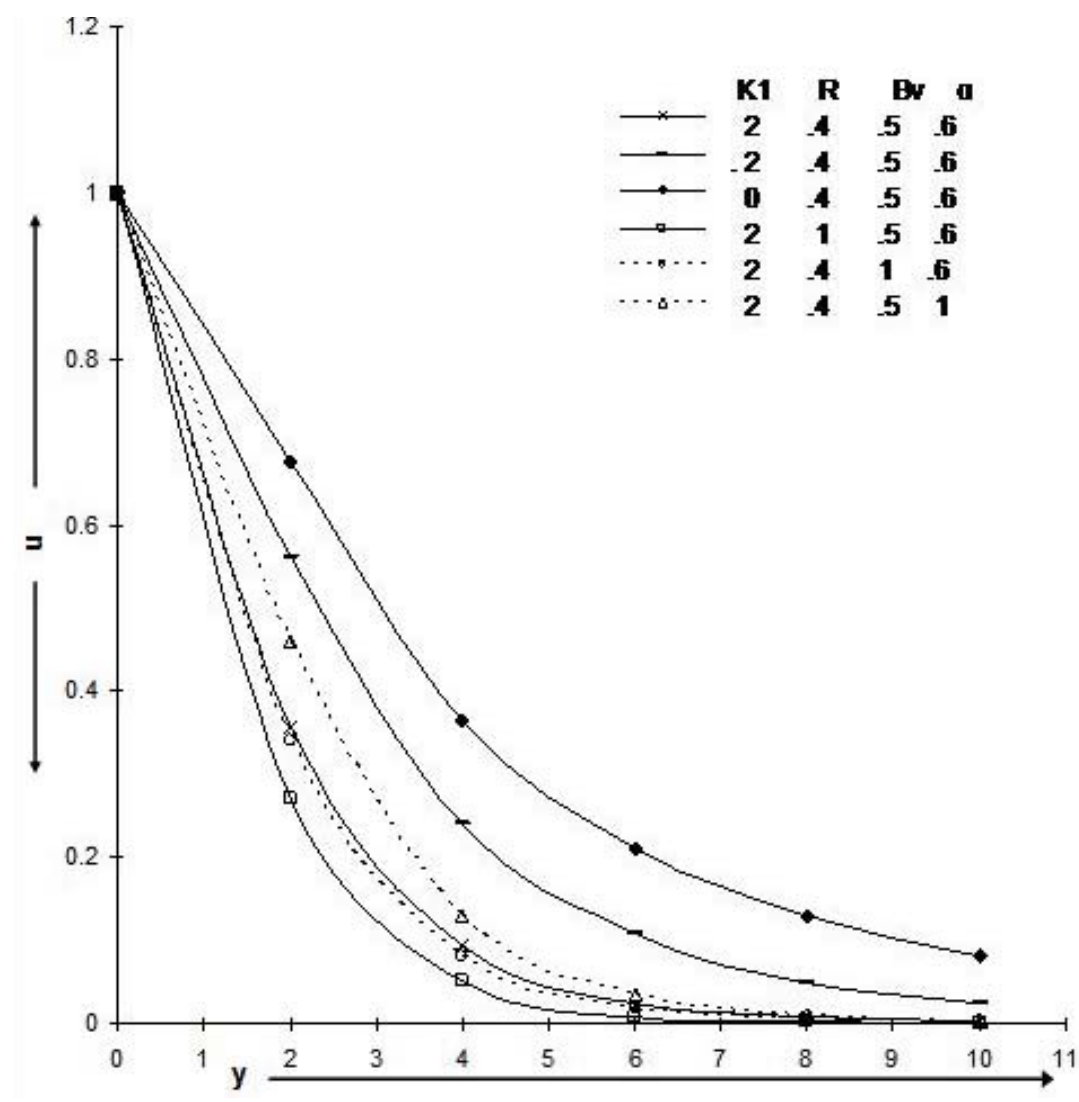

Fig.5. Velocity profiles $u$ for $\operatorname{Pr}=1, M=1, \mathrm{Gr}=2, S=1, m=0.5, \mathrm{Sc}=0.22, B=0.5$. 


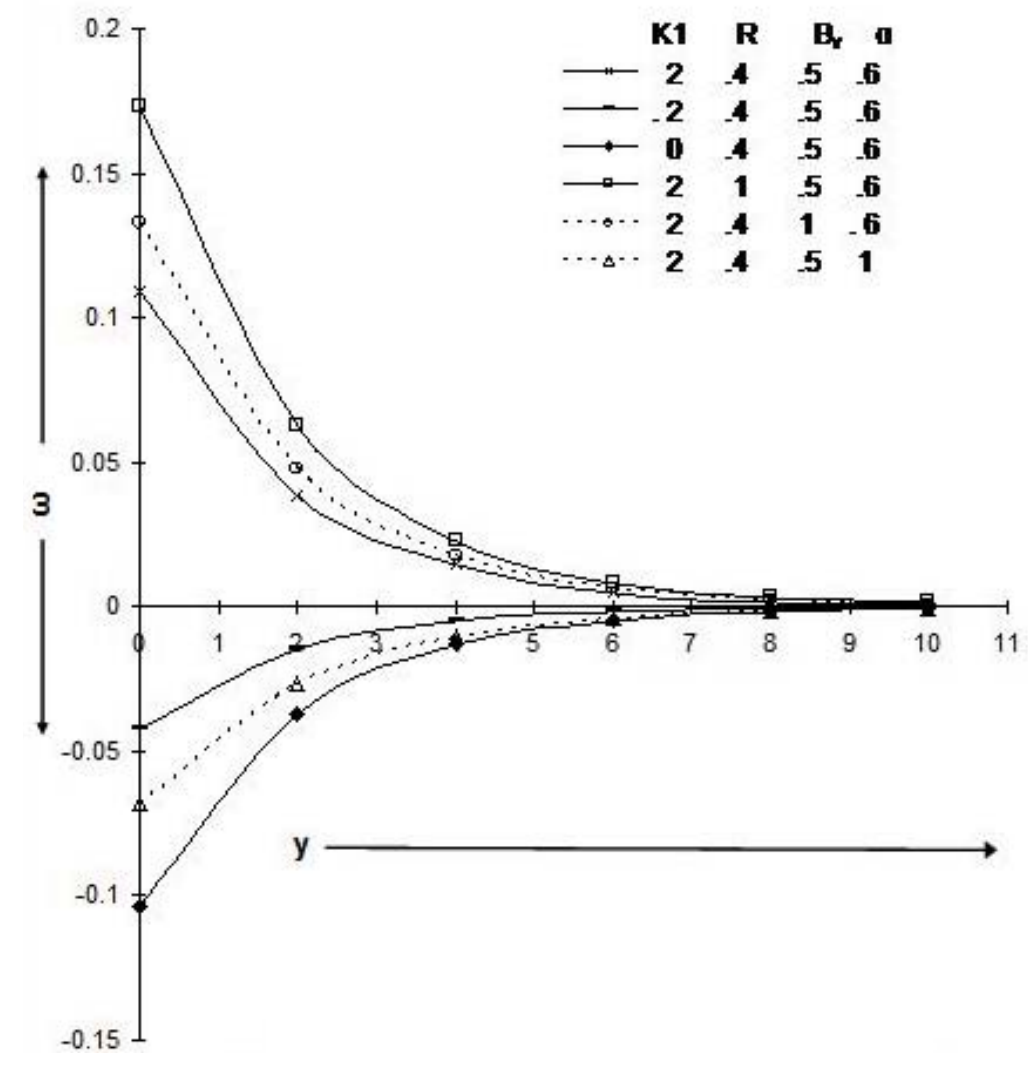

Fig.6. Microrotation profiles $\omega$ for $\operatorname{Pr}=1, M=1, \mathrm{Gr}=2, S=1, m=0.5, \mathrm{Sc}=0.22, B=0.5$.

Figures 7 and 8 illustrate the translational velocity and microrotation profiles against the span-wise coordinate $y$ for various values of the Prandtl number (Pr), Grashof number (Gr), magnetic parameter $(M)$, heat source parameter $(S), \mathrm{m}$ and Schmidt number $(\mathrm{Sc})$, respectively. It is observed that, keeping other parameters fixed, as the magnetic parameter increases, the translational velocity $u$ decreases. The presence of a magnetic field in an electrically conducting fluid introduces a force called Lorentz force which acts against the flow if the magnetic field is applied in the normal direction as considered in the present problem. This type of resistive force tends to slow down the flow field. It is clear that there exists an overshooting of the velocity $u$ for small values of $M$ (e.g.; $M=1.0$ ). Since the magnetic field has a stabilizing effect, the velocity overshoot decreases with increasing $M$ and vanishes for higher values of $M$ (e.g.; $M=3$ ). It is noticed that an increase in Gr leads to a rise in the values of velocity $u$ because the favorable buoyancy force accelerates the fluid, while a reverse effect is noted for the magnitude of microrotation component $\omega$. Furthermore, it is seen that the effect of increasing values of Sc results in decreasing $u$, while an opposite behavior is observed for microrotation $\omega$ across the boundary layer. It is reported that as the coefficient $m$ increases, the translational velocity and the magnitude of microrotation profiles increase. Furthermore, as expected, the translational velocity and the magnitude of microrotation at the wall decrease on increasing the values of $\operatorname{Pr}$ and $S$. 


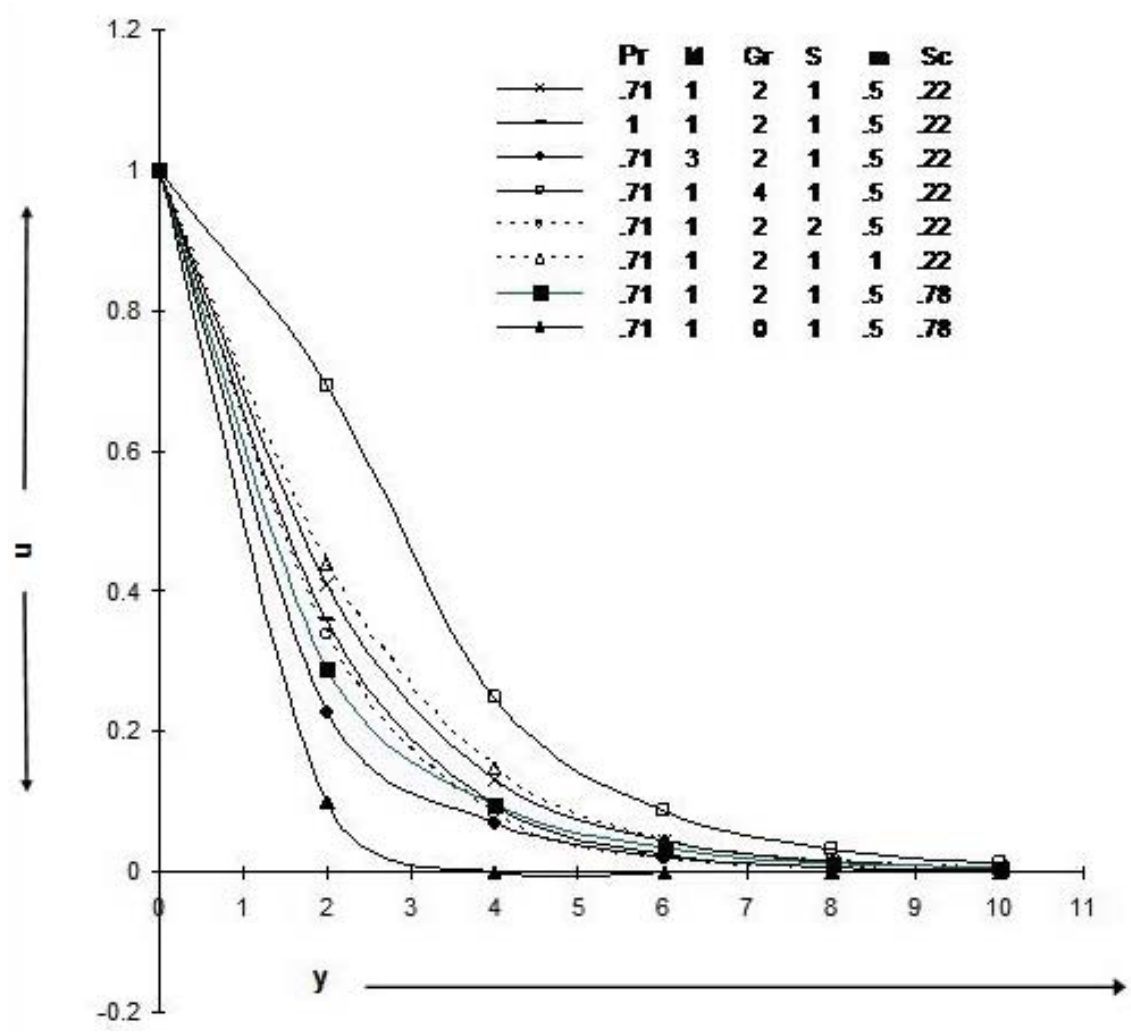

Fig.7. Velocity profiles $u$ for $K 1=2, B=0, B_{v}=0.5, \alpha=0.6$.

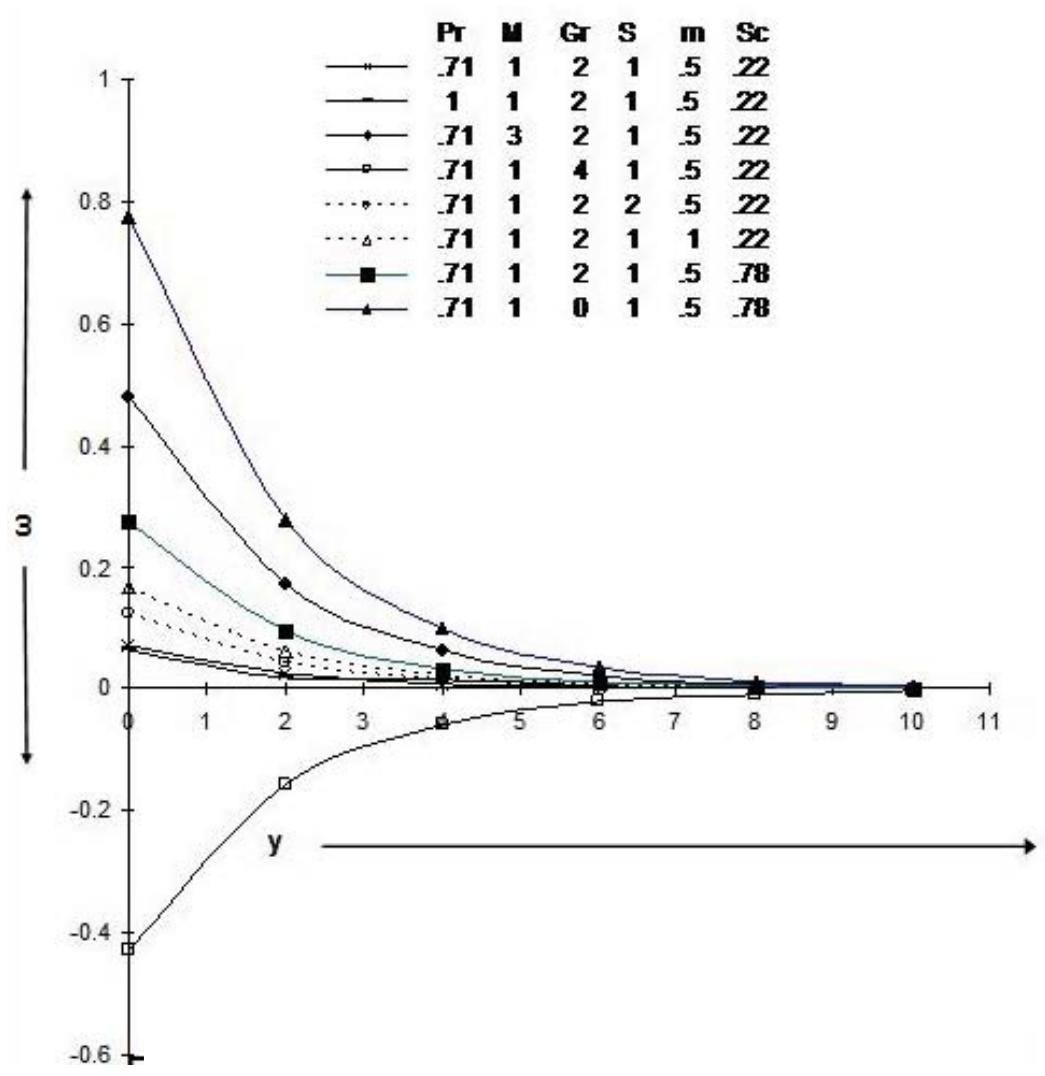

Fig.8. Microrotation profiles $\omega$ for $K 1=2, R=0.4, B_{v}=0.5, B=0.5, \alpha=0.6$. 
Given the velocity field in the boundary layer, the skin friction coefficient $\left(C_{f}\right)$ at the wall can be calculated, which is given by

$$
\begin{aligned}
& \tau_{\omega}=\left.(\mu+\Lambda) \frac{\partial u^{*}}{\partial y^{*}}\right|_{y^{*}=0}+\left.\Lambda \omega^{*}\right|_{y^{*}=0}, \\
& C_{f}=\frac{2 T_{w}^{*}}{\rho U_{0} V_{0}}=2[1+(1-n) \beta] u^{\prime}(0) .
\end{aligned}
$$

The couple stress coefficient $\left(C_{m}\right)$ at the plate is written as

$$
\begin{aligned}
& C_{\omega}=\left.\gamma \frac{\partial \omega^{*}}{\partial y^{*}}\right|_{y^{*}=0}, \\
& C_{m}=\frac{M_{w}}{\mu J U_{0}}=\left(1+\frac{\beta}{2}\right) w^{\prime}(0) .
\end{aligned}
$$

Figures 9 and 10 show the variations of the skin friction coefficient $C_{f}$ and couple stress coefficient $C_{m}$ for various values of $K_{l}, R, B_{v}$ and $\alpha$ against Gr. It is observed that higher values of the radiation parameter and chemical reaction parameter lead to a reduction in the skin friction coefficient and couple stress coefficient. It is noted that $C_{f}$ and $C_{m}$ decrease with increasing $B_{v}$, because increasing $B_{v}$ offers a greater resistance to the fluid motion. It is concluded that $C_{f}$ and $C_{m}$ increase with increasing the values of $\alpha$. Further, $C_{f}$ and $C_{m}$ increase as the buoyancy parameter $\mathrm{Gr}$ increases. The reason for this is that an increase in the buoyancy effect in a mixed convection flow leads to an acceleration of the fluid flow, which increases the friction factor.

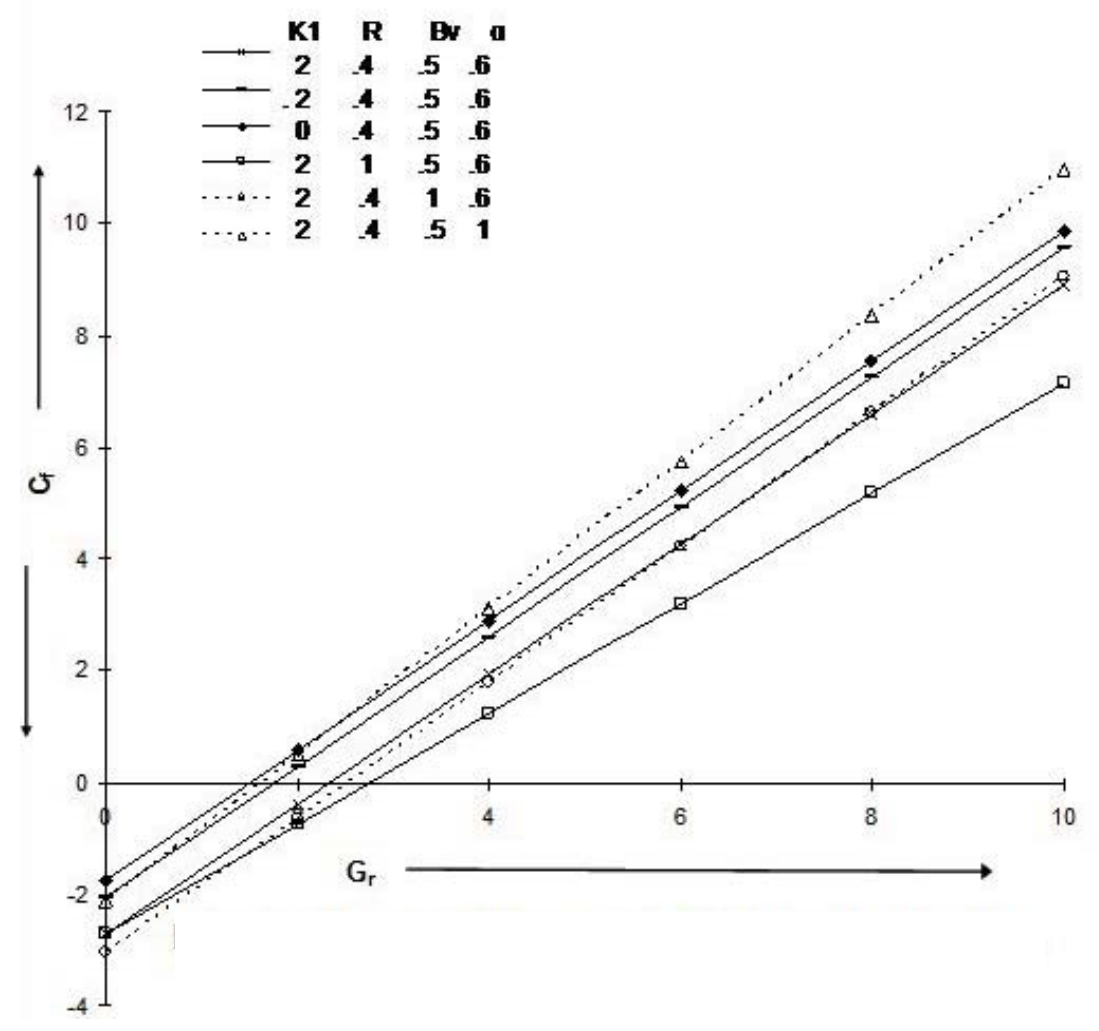

Fig.9. Skin-friction coefficient for $\operatorname{Pr}=1, M=1, S=1, m=0.5, \mathrm{Sc}=0.22, B=0.1$. 


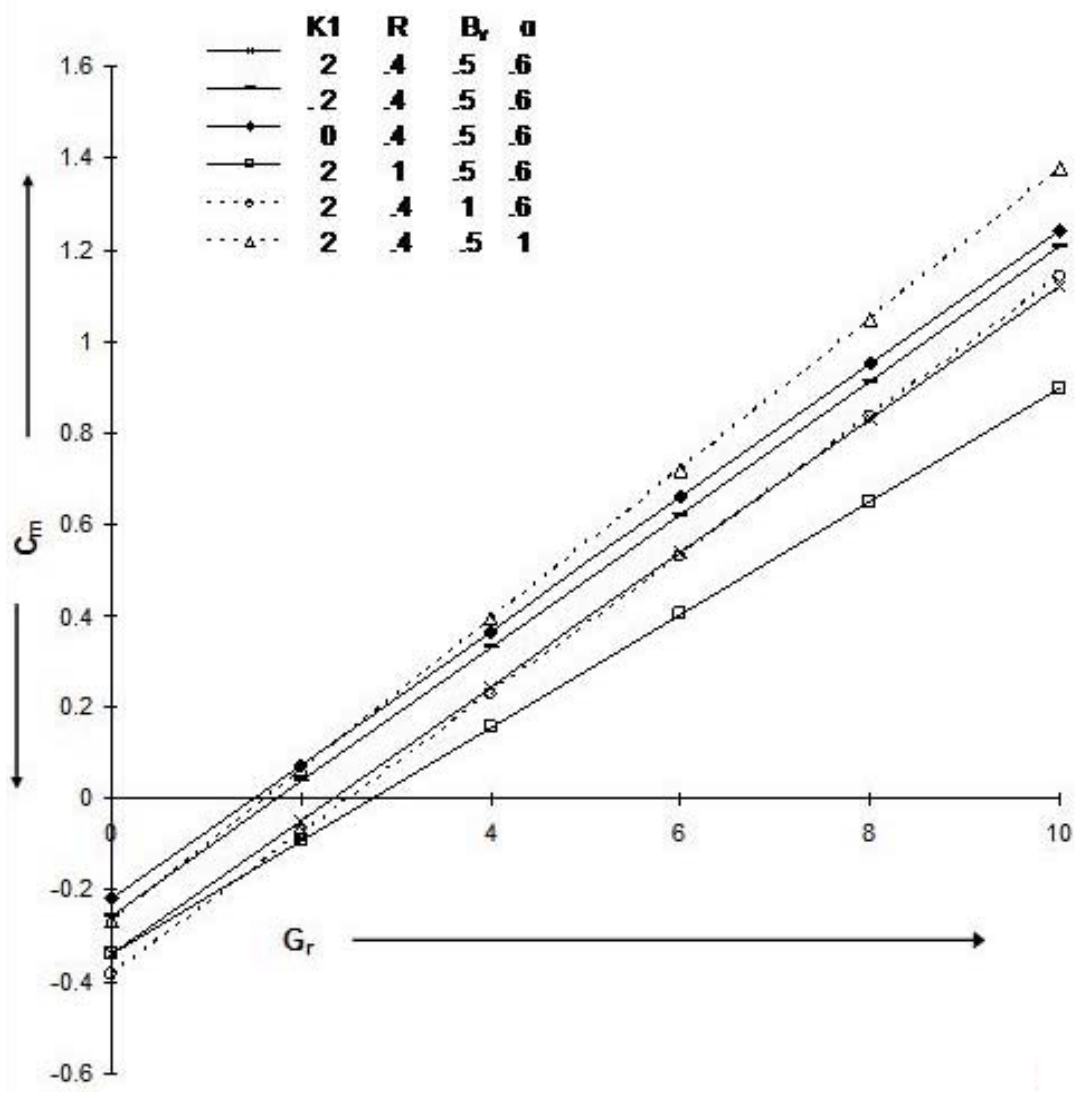

Fig.10. Couple stress coefficient for $\operatorname{Pr}=1, M=1, S=1, m=0.5, \mathrm{Sc}=0.22, B=0.1$.

The variations of the skin friction coefficient $C_{f}$ and couple stress coefficient $C_{m}$ for various values of $\operatorname{Pr}, M, S, \mathrm{Sc}, B_{v}$ and $\mathrm{m}$ are shown in Figs 11 and 12, respectively. It is found that $C_{f}$ is lower for micropolar fluids $\left(B_{v} \neq 0\right)$ than for Newtonian fluids $\left(B_{v}=0\right)$. Such a result is expected since micropolar fluids offer a greater resistance (resulting from dynamic viscosity $(\mu)$ and vortex viscosity $(\Lambda)$ ) to the fluid motion compared with Newtonian fluids. It is noted that the skin friction and the wall couple stress decrease as $M, S$, $\mathrm{Sc}$, and $m$ increase. It is deduced that $C_{f}$ and $C_{m}$ decrease as Pr increases. Physically, this is true because the increase in $\mathrm{Pr}$ is due to an increase in the viscosity of the fluid, which makes the fluid thick and hence a decrease in the velocity of the fluid. 


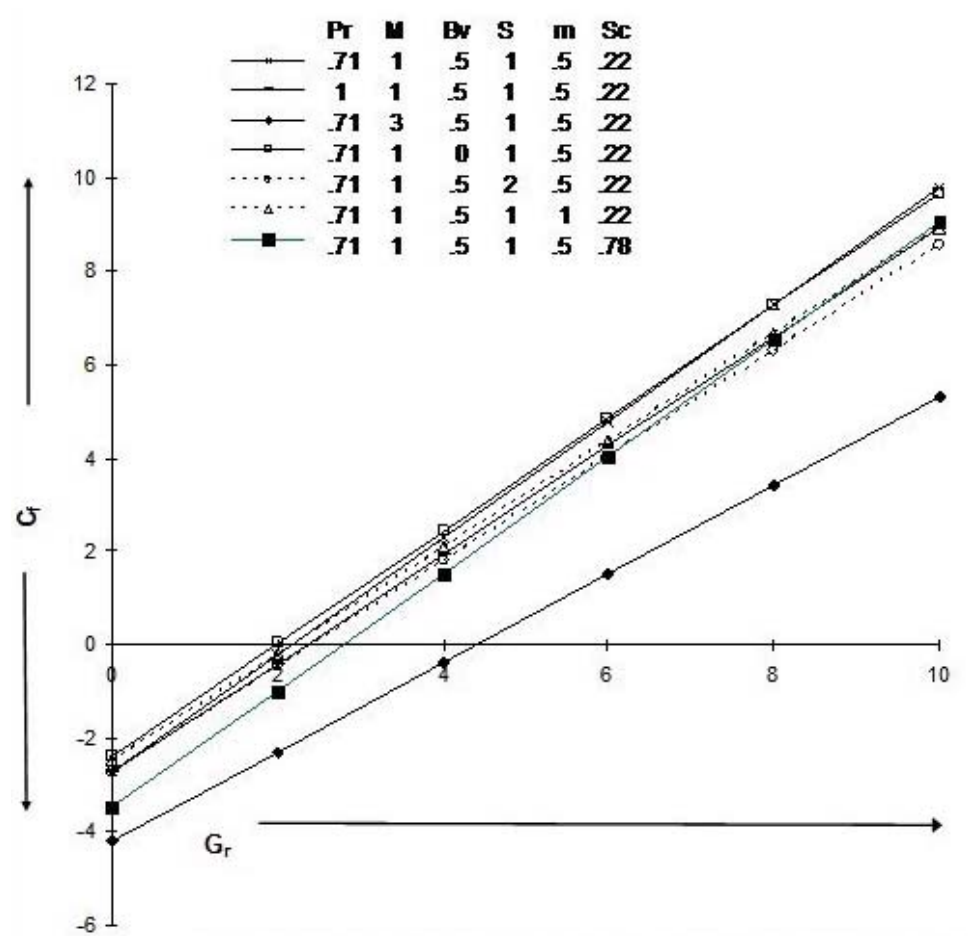

Fig.11. Skin-friction coefficient for $K 1=2, R=0.4, B=0.1, \alpha=0.6$.

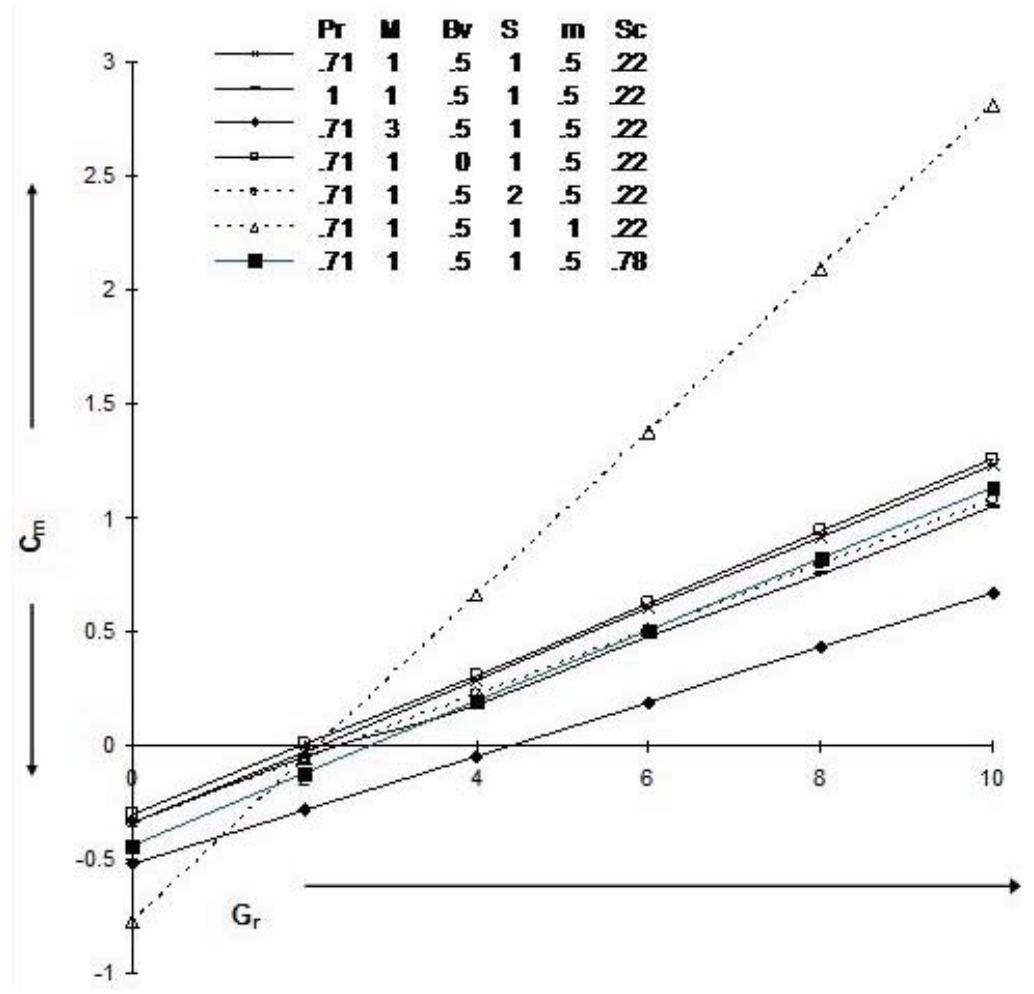

Fig.12. Couple stress coefficient for $K 1=2, R=0.4, B=0.1, \alpha=0.6$.

The rate of heat transfer in terms of the Nusselt number is given by 


$$
\mathrm{Nu}=\frac{q_{w}^{*} v}{\kappa U_{0}^{*}\left(T_{w}^{*}-T_{\infty}^{*}\right)}
$$

where $\quad \mathrm{q}=-\left.\kappa \frac{\partial T^{*}}{\partial y^{*}}\right|_{y^{*}=0}$,

in a non-dimensional form it is given by

$$
\mathrm{Nu}=-\left.\frac{\partial \theta}{\partial y}\right|_{y=0}
$$

The rate of mass transfer is given by

$$
J^{*}(\text { Diffusion flux })=-\left.\rho D^{*} \frac{\partial C^{*}}{\partial y^{*}}\right|_{y^{*}=0} .
$$

The coefficient of mass transfer which is generally known as the Sherwood number $S_{h}$ is given by

$$
\mathrm{S}_{\mathrm{h}}=\frac{J^{*} v}{V_{0} \rho D^{*}\left(C_{w}^{*}-C_{\infty}^{*}\right)}=-\left.\frac{\partial C}{\partial y}\right|_{y=0} .
$$

Numerical values of the rate of heat transfer for selected values of the Prandtl number (Pr), radiation parameter $(R)$ and heat source parameter $(S)$ taking $\varepsilon=0.01, n=1$ and $t=1$ are presented in Fig. 13. It is important to note that the heat transfer rate is enhanced as the Prandtl number and radiation parameter increase. This is due to the fact that there would be a decrease of thermal boundary layer thickness with the increase of the Prandtl number. It is also observed that the Nusselt number increases with an increase in $S$ or $R$.

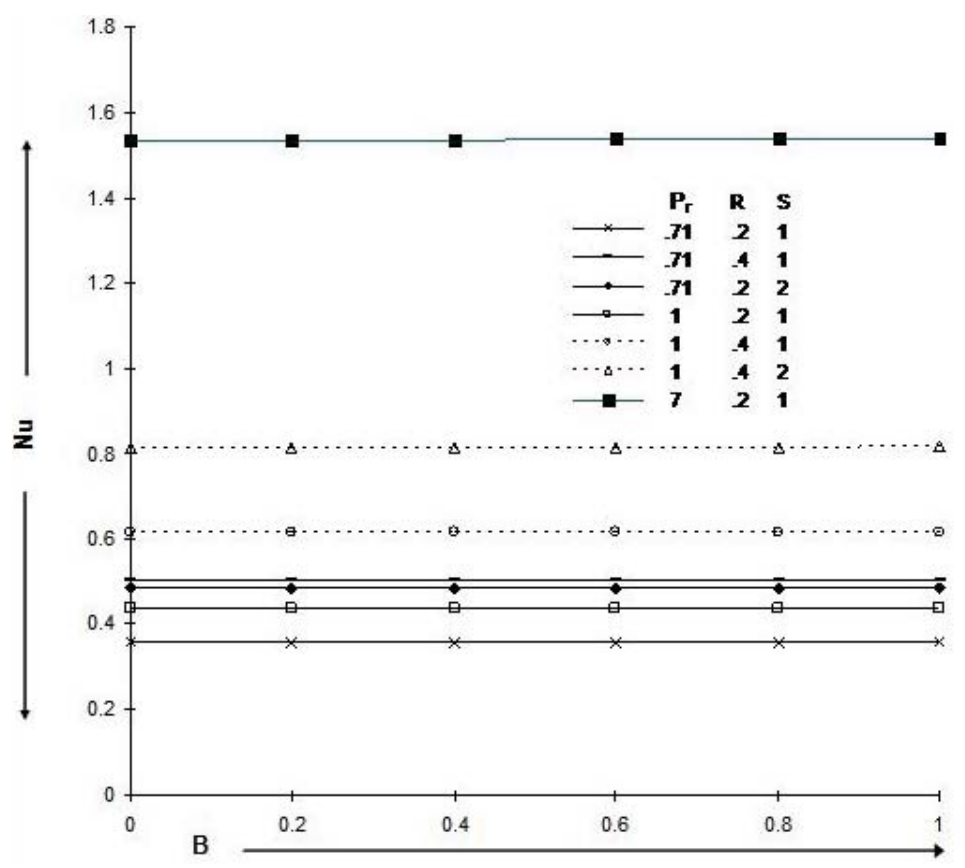

Fig.13. Rate of heat transfer $\varepsilon=0.01, n=0.1, t=1$. 
Figure 14 displays the rate of mass transfer at the porous plate for hydrogen $(\mathrm{Sc}=0.22)$, oxygen $(\mathrm{Sc}$ $=0.66)$ and ammonia $(\mathrm{Sc}=0.78)$ at temperature $25^{\circ} \mathrm{C}$ and 1 atmospheric pressure taking $\varepsilon=0.01, n=1$ and $t$ $=1$. It is clear that increasing the values of the Schmidt number or chemical reaction parameter enhance the mass transfer rate. Physically, the increase of Sc means a decrease of molecular diffusivity (D), that results in a decrease of concentration boundary layer. Hence, the concentration of the species is higher for values of Sc and lower for larger values of Sc.

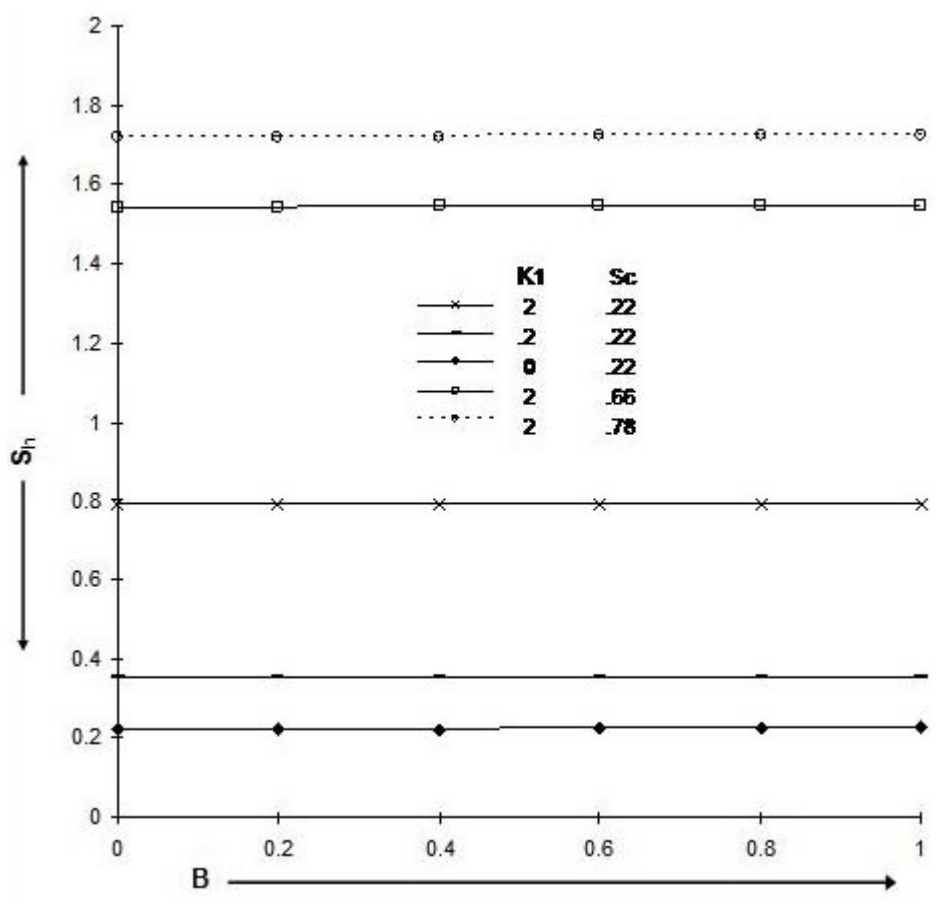

Fig.14. Rate of mass transfer $\varepsilon=0.01, n=0.1, t=1$.

\section{Nomenclature}

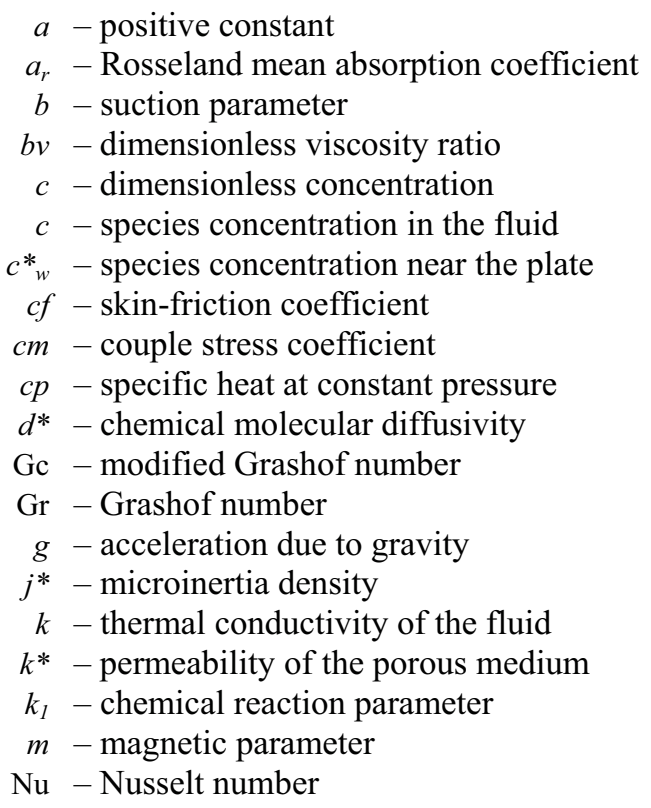




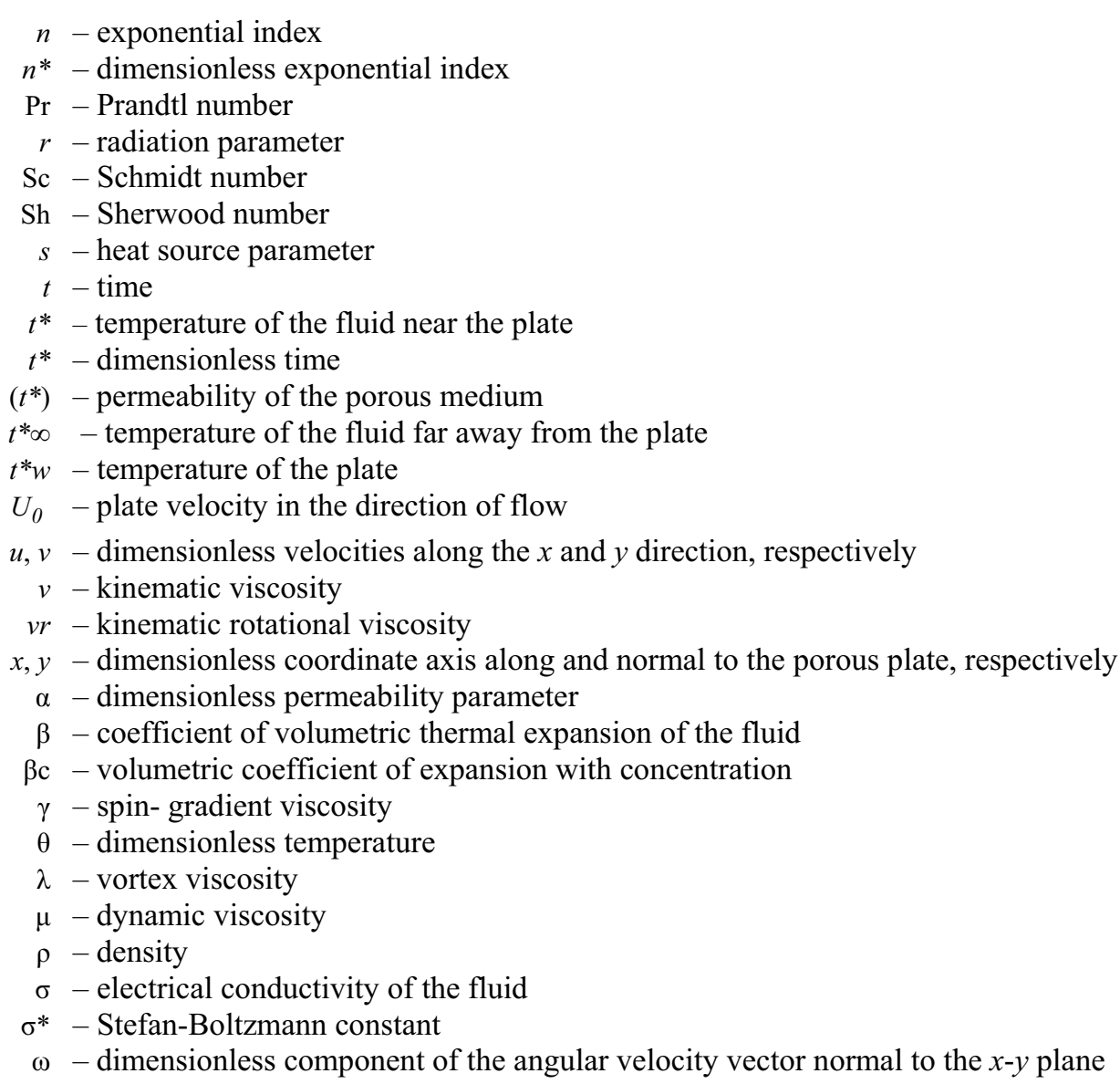

\section{Subscripts}

$w$ - wall condition

$\infty-$ free stream condition

\section{Superscripts}

$*-$ dimensional properties

\section{References}

Abd-ElAziz M. (2006): Thermal radiation effects on magnetohydro-dynamicmixed convection flow of a micropolar fluid past a continuously moving semi-infinite plate for high temperature differences. - Acta Mechanica, vol.187, pp.113-127.

Ariman T., Turk M.A. and Sylvester N.D. (1973): Microcontinuum fluid mechanics - a review. - Int. J. Engng. Sci., vol.11, pp.905-930.

Baehr H.D. and Stephan K. (1998): Heat and Mass Transfer. - Berlin Heidelberg: Springer-Verlag.

Chaudhary R.C. and Jain P. (2007): Combined heat and mass transfer in magneto-micropolar fluid flow from radiative surface with variable permeability. - ZAMM, vol.87, pp.(8-9), 549-563.

Chaudhary R.C. and Sharma B.K. (2006): Combined heat and mass transfer by laminar mixed convection flow from a vertical surface with induced magnetic field. - Journal of Applied Physics, vol.99, pp.34901-10.

Cussler E.L. (1998): Diffusion Mass Transfer in Fluid Systems. - $2^{\text {nd }}$ ed. Cambridge University Press. 
Das U.N., Deka R.K. and Soundalgekar V.M. (1994): Effects of mass transfer on flow past an impulsively started infinite vertical plate with constant heat flux and chemical reaction. - Forschung im Engenieurwesen Engineering Research Co., pp.284-287.

El-Amin M.F. (2001): Magnetohydrodynamic free convection and mass transfer flow in micropolar fluid with constant suction. - J. Magn. Mater., vol.234, pp.567-574.

Elbashbeshy E.M.A. and Bazid M.A.A. (2000): Effect of radiation on forced convection flow of a micropolar fluid over a horizontal plate. - Can. J. Phys./Rev. Can. Phys., vol.78 (10), pp.907-913.

El-Hakiem M.A., Mohammadein A.A., El-Kabeir S.M.M. and Gorla R.S.R. (1999): Joule heating effects on magnetohydrodynamic free convection flow of a micropolar fluid. - Int. Comm. Heat Mass Tran., vol.26(2), pp.219227.

Eringen A.C. (1964): Simple microfluids. - Int. J. Engng. Sci., vol.2, pp.205-217.

Eringen A.C. (1966): Theory of micropolar fluids. - J. Math. Mech., vol.16, pp.1-18.

Eringen A.C. (1972): Theory of termomicrofluids. - J. Math. Anal. Appl., vol.38, pp.480-496.

Gorla R.S.R. (1992): Mixed convection in a micropolar fluid from a vertical surface with uniform heat flux. - Int. J. Engng. Sci., vol.30, pp.349-358.

Gorla R.S.R., Mohammedan A., Mansour M. and Hussein I. (1995): Unsteady natural convection from a heated vertical plate in micropolar fluid. - Numerical Heat Transfer, Part A, vol.28, pp.253-262.

Gorla R.S.R., Slaouti A.S. and Takhar H.S. (1998): Free convection in micropolar fluids over a uniformly heated vertical plate. - Int. J. Heat and Fluid Flow., vol.8, pp.504-518.

Helmy K.A. (1998): MHD unsteady free convection flow past a vertical porous plate. - ZAMM, vol.98, pp.255-270.

Helmy K.A., Idriss H.F. and Kassem S.E. (2002): MHD free convection flow of a micropolar fluid past a vertical porous plate. - Can. J. Phys./Rev. Can. Phys., vol.80 (12), pp.1661-1673.

Hiremath P.S. and Patil P.M. (1993): Free convection effects on oscillatory flow of couple stress field through a porous medium. - Acta Mech., vol.98, pp.143-158.

Ibrahim F.S., Hassanien I.A. and Bakr A.A. (2004): Unsteady magneto-hydrodynamic micropolar fluid flow and heat transfer over a vertical porous plate through a porous medium in the presence of thermal and mass diffusion with a constant heat source. - Can. J. Phys./Rev. Can. Phys., vol.82 (10), pp.775-790.

Kandasamy R., Periasamy K. and Prashu Sivagnana K.K. (2005): Effects of chemical reaction, heat and mass transfer along wedge with heat source and concentration in the presence of suction or injection. - Int. J. Heat Mass Transfer, vol.48, pp.1388-1394.

Kim Y.J. (2001): Unsteady convection flow of micropolar fluids past a vertical plate embedded in a porous medium. Acta. Mech., vol.148, pp.105-116.

Kim Y.J. (2004): Heat and mass transfer in MHD micropolar flow over a vertical moving plate in a porous medium. Trans. Porous Media, vol.56, pp.17-37.

Kim Y.J. and Fedorov A.G. (2003): Transient mixed radiative convection flow of a micropolar fluid past a moving semi-infinite vertical porous plate. - Int. J. Heat Mass Transfer, vol.46 (10), pp.1751-1758.

Kim Y.J. and Lee J.C. (2003): Analytical studies on MHD oscillatory flow of a micropolar fluid over a vertical porous plate. - Surface and Coatings Technology, vol.171, pp.187-193.

Lukaszewicz G. (1999): Micropolar Fluids. - Theory and Applications, Boston: Birkhaüser.

Muthucumarswamy R. (2002): Effects of a chemical reaction on moving isothermal vertical surface with suction. Acta. Mech., vol.155, pp.65-70.

Muthucumarswamy R. and Ganesan P. (2001): First order chemical reaction on flow past an impulsively started vertical plate with uniform heat and mass flux. - Acta. Mech., vol.147, pp.45-57.

Perdikis C. and Raptis A. (1996): Heat transfer of a micropolar fluid by the presence of radiation. - Heat and Mass Transfer, vol.31 (6), pp.381-382. 
Perdikis C. and Raptis E. (2006): Unsteady MHD flow in the presence of radiation. - Int. J. of Applied Mechanics and Engineering, vol.11 (2), pp.383-390.

Rahman M.M. and Sattar M.A. (2006): Magneto-hydrodynamic convective flow of a micropolar fluid past a continuously moving vertical porous plate in the presence of heat generation/absorption. - ASME J. Heat Transfer, vol.128 (2), pp.142-152.

Raptis A. (1998): Flow of a micropolar fluid past a continuously moving plate by the presence of radiation. - Int. J. Heat Mass Transfer, vol.41, pp.2865-2866.

Raptis A. and Massalas C.V. (1998): Magnetohydrodynamic flow past a plate by the presence of radiation. - Heat and Mass Transfer, vol.34, pp.107-109.

Raptis A. and Takhar H.S. (1999): Polar fluid through a porous medium. - Acta Mechanica, vol.135, pp.91-93.

Raptis A. Perdikis C. and Angew Z. (1998): Viscoelastic flow by the presence of radiation. - Math. Mech., vol.78 (4), pp.277-279.

Rees D.A.S. and Pop I. (1998): Free convection boundary layer flow of a micropolar fluid from a vertical flat plate. IMAJ. Appl. Math., vol.61, pp.179-197.

Sharma B.K. and Chaudhary R.C. (2008): Hydromagnetic unsteady mixed convection and mass transfer flow past a vertical porous plate immersed in a porous medium with hall effect. - Engineering Transactions, vol.56(1), pp.3-23.

Sharma B.K., Agarwal M. and Chaudhary R.C. (2006): MHD fluctuating free convective flow with radiation embedded in porous medium having variable permeability and heat source/sink. - Journal of Technical Physics, vol.47(1), pp.47-58.

Sharma B.K., Chaudhary R.C. and Agarwal M. (2007): Radiation effect on temperature distribution in threedimensional Couette flow with injection or suction. - Applied Mathematics and Mechanics, vol.28(3), pp.309-316.

Sharma B.K., Chaudhary R.C. and Agarwal M. (2008): Radiation effect on steady free convective flow along a uniform moving porous vertical plate in presence of heat source/sink and transverse magnetic Field. - Bull. Cal. Math. Soc., vol.100, pp.529-538.

Sharma B.K., Chaudhary R.C. and Sharma P.K.(2007): Fluctuating mass transfer on three-dimensional flow through a porous medium with variable permeability. - Advances in Theoretical and Applied Mathematics., vol.2(3), pp.257267.

Sharma B.K., Jha A.K. and Chaudhary R.C. (2006): Radiation effect with simultaneous thermal and mass diffusion in MHD mixed convection flow from a vertical surface with ohmic heating. - Romania Journal of Physics, vol.51(7-8), pp.715-727.

Soundalgekar V.M. and Takhar H.S. (1983): Flow of micropolar fluid past a continuouslymoving plate. - Int. J. Eng. Sci., vol.21, pp.961-965.

Takhar H.S. and Soundalgekar V.M. (1980): Heat transfer in a flat plate flow of a micropolar fluid. - Rheologica Acta, vol.19, pp.525-526.

Takhar H.S. and Soundalgekar V.M. (1985): Flow and heat transfer of micropolar fluid past a porous plate. - Indian J. Pure Appl. Math., vol.16, pp.552-558.

Takhar H.S. and Soundalgekar V.M. (1985): Flow and heat transfer of a micropolar fluid past a continuously moving porous plate. - Int. J. Eng. Sci., vol.23, pp.201-205.

Yucel A. (1989): Mixed convection in micropolar flow over a horizontal plate with surface mass transfer. - Int. J. Eng. Sci., vol.27, pp.1593-1602. 